\title{
Clear Cell Sarcoma Shows Immunoreactivity for Microphthalmia Transcription Factor: Further Evidence for Melanocytic Differentiation
}

Scott R. Granter, M.D., Katherine N. Weilbaecher, M.D., Catherine Quigley, FIBMS, Christopher D.M. Fletcher, M.D, F.R.C.Path, David E. Fisher, M.D. PhD.

Department of Pathology (SRG, CDMF, CQ), Brigham and Women's Hospital and Harvard Medical School, Boston, Massachusetts; Division of Pediatric Oncology (KNW), Dana Farber Cancer Institute and Children's Hospital, Boston, Massachusetts (KNW, DEF)

Microphthalmia transcription factor, a melanocytic nuclear protein critical for the embryonic development and postnatal viability of melanocytes, is a master regulator in modulating extracellular signals. Recently, microphthalmia transcription factor expression was shown to be both a sensitive and specific marker of epithelioid melanoma. We investigated the sensitivity of D5, an anti-microphthalmia transcription factor antibody, for diagnosis of clear cell sarcoma (also known as malignant melanoma of soft parts). Immunoreactivity in a nuclear pattern for D5 was present in 8 of 12 (75\%) tumors. D5 staining was strong in three tumors, moderate in two, and weak in three. S-100 protein expression was seen in all 12 cases that had clear cell sarcoma examined. HMB-45 staining was seen in 11 of 12 $(92 \%)$ tumors. Focal Melan-A positivity was seen in 3 of 7 (43\%) tumors. Although D5 was shown in a previous study to be a highly sensitive and specific marker for epithelioid melanomas, the results of this study expand the spectrum of tumors showing immunoreactivity for D5. D5 immunoreactivity in clear cell sarcoma provides further evidence for melanocytic differentiation in this unusual tumor.

KEY WORDS: Clear cell sarcoma, D5 immunoreactivity, Microphthalmia transcription factor, melanoma.

Mod Pathol 2001;14(1):6-9

Clear cell sarcoma is an unusual and rare tumor that most often presents on the distal extremities of adolescents and young adults $(1,2)$. Most tumors involve tendon, fascia, or aponeuroses. They are

Copyright (C) 2001 by The United States and Canadian Academy of Pathology, Inc.

VOL. 14, NO. 1, P. 6, 2001 Printed in the U.S.A.

Date of acceptance: August 29, 2000.

Address reprint requests to: Scott R. Granter, M.D., Department of Pathology, Brigham and Women's Hospital, 75 Francis Street, Boston, MA 02115; fax: 617-739-6192. aggressive tumors with a propensity for metastases that may be delayed for many years after diagnosis. Several lines of evidence for melanocytic differentiation in clear cell sarcoma have been demonstrated. Approximately two-thirds of tumors contain melanin pigment visible on routine histologic sections. Immunoreactivity for $\mathrm{HMB}-45$, a relatively specific melanoma marker, is present in most cases. Ultrastructural examination usually demonstrates melanosomes (3-5). Although many of the above features point to melanocytic differentiation, clear cell sarcoma is a distinctive tumor rather than simply a deep form of malignant melanoma. Characteristic clinical and histopathologic features argue that clear cell sarcoma is a unique entity. Perhaps the most compelling evidence, however, is the demonstration in the majority of clear cell sarcomas of a consistent translocation between chromosome 12 and 22 that is not seen in conventional malignant melanoma (6-8). This translocation results in fusion of the EWS (Ewing's sarcoma) gene and ATF-1 (9).

Often it is difficult to distinguish large, deeply invasive spindle cell melanomas that lack a demonstrable in situ component from clear cell sarcoma on the basis of histologic examination. Unfortunately, there are currently no immunomarkers that allow distinction between clear cell sarcoma and conventional melanoma. In this study, we examined the immunoreactivity of clear cell sarcoma for microphthalmia transcription factor (Mitf). Mitf, the protein encoded by the microphthalmia ( $\mathrm{mi}$ ) gene, has been shown to be essential for the development and survival of melanocytes. Recently, D5, a monoclonal antibody generated against human Mitf, was shown to be reactive in $100 \%$ of epithelioid melanomas studied (10). Herein, we report our experience studying clear cell sarcoma by use of the D5 antibody. 


\section{MATERIALS AND METHODS}

Twelve cases of clear cell sarcoma were retrieved from the authors' consultation files (CDMF) and the files of Brigham and Women's Hospital. Inclusion in the study required that the tumor have characteristic histologic features of clear cell sarcoma without any evidence of in situ melanoma. In one patient, tissue from a lung metastasis with a primary clear cell sarcoma of the finger was used for immunostaining. In all other patients, tissue from the primary tumor was used for immunostaining. Immunohistochemical studies were performed on all cases using formalin-fixed, paraffinembedded tissue. Sections were cut at $4 \mu \mathrm{m}$ and dried at $37^{\circ} \mathrm{C}$. Paraffin was removed by xylene, and the sections were hydrated in a graded series of alcohols. Primary antibodies included polyclonal rabbit antibody S-100 protein (DAKO, Carpenteria, CA) at 1:5000 dilution, mouse monoclonal antibody HMB-45 (DAKO) at 1:400 dilution, Melan-A (DAKO) at 1:200 dilution, and undiluted mouse monoclonal antibody D5 (tissue culture supernatant) (10). No pretreatment was used for S-100 and HMB-45 immunostains.

Microwave antigen retrieval for D5 and Melan-A immunostains was performed with $10 \mathrm{~mm}$ citrate buffer at pH 6.0 for 30 minutes at $92.8^{\circ} \mathrm{C}$; next was a 10-minute cooldown period. All staining was performed with DAKO Envision+ detection system and 3,3'-diaminobenzidine + as the chromogen. Melanomas known to express the antigen of interest were used as positive controls, whereas removal of the primary antibodies in the test tissues was used as negative controls. Only a nuclear pattern of D5 staining was regarded as positive. HMB-45 antibody staining was considered positive if cytoplasmic staining was present. S-100 protein was considered positive if there was both a cytoplasmic and nuclear reaction. All staining (with the exception of samples from one patient, whose slides were no longer available for scoring) was quantitated as fo- cal or weak staining $(+)$ in rare cells (less than 5\%), moderate staining with strong positivity in 5 to $50 \%$ of cells $(++)$, and diffuse strong staining $(+++)$ with more than $50 \%$ of cells immunoreactive.

\section{RESULTS}

Table 1 summarizes the clinical features of the 12 clear cell sarcomas we studied. As is characteristic of clear cell sarcoma, most of the patients' sarcomas arose on the distal extremities, particularly near joints. Also characteristic of clear cell sarcoma is the young age of the patients studied (median age, 28.5 y). All patients showed classic histomorphologic features (Fig. 1).

Table 1 summarizes the results of studies with S-100 protein, HMB-45, Melan-A, and D5 immunostains. In one case, tissue from a pulmonary metastasis in a patient with a primary finger tumor was used for immunostudies. In all but one case, immunoreactivity was scored as focal, moderate, or strong, as described above. Immunoreactivity in a nuclear pattern for D5 was present in 8 of 12 tumors (Fig. 2). D5 staining was strong in three tumors, moderate in two, and weak in three. Nonspecific faint cytoplasmic blush was seen in several tumors; S-100 protein expression was seen in all 12 tumors; focal staining was seen in 6 of 11 tumors; and moderate staining was present in 3 of 11. Strong staining for S-100 was seen in only 2 of 11 tumors. HMB- 45 staining was seen in 11 of 12 tumors. Staining for HMB-45 was strong in 5 of 11 tumors, moderate in 4 of 11 tumors and weak in 1 patient. Melan-A immunostaining was performed in seven tumors. Focal Melan-A positivity was seen in three tumors all of which were reactive for D5.

\section{DISCUSSION}

Clear cell sarcoma is an uncommon tumor of uncertain histogenesis that most often arises in as-

TABLE 1. Clear Cell Sarcomas: Clinical and Immunohistochemical Findings

\begin{tabular}{|c|c|c|c|c|c|c|c|}
\hline Patient & Age & Sex & Site & S-100 & HMB-45 & D5 & Melan-A \\
\hline 1 & 28 & M & Finger & + & ++ & +++ & - \\
\hline 2 & 15 & M & Pelvis & ++ & +++ & ++ & \\
\hline 3 & 43 & M & Foot & +++ & +++ & + & + \\
\hline 4 & 9 & M & Lower back & + & +++ & ++ & + \\
\hline 5 & 22 & $\mathrm{~F}$ & Knee & +++ & + & +++ & \\
\hline 6 & 19 & $\mathrm{~F}$ & Calf & ++ & ++ & + & + \\
\hline 7 & 33 & $\mathrm{~F}$ & Hand & + & ++ & +++ & - \\
\hline 8 & 33 & $\mathrm{M}$ & Thigh & + & - & - & - \\
\hline 9 & 24 & $\mathrm{M}$ & Foot & ++ & +++ & + & - \\
\hline 10 & 36 & M & Triceps tendon & + & +++ & - & \\
\hline 11 & 45 & $\mathrm{M}$ & $\begin{array}{l}\text { Pulmonary metastasis } \\
\text { from finger }\end{array}$ & + & ++ & - & \\
\hline 12 & 34 & $\mathrm{~F}$ & Popliteal fossa & $+^{*}$ & $+^{*}$ & - & \\
\hline
\end{tabular}

D, desmoplastic melanoma; F, female; M, male.

* Immunoresults not quantified. 


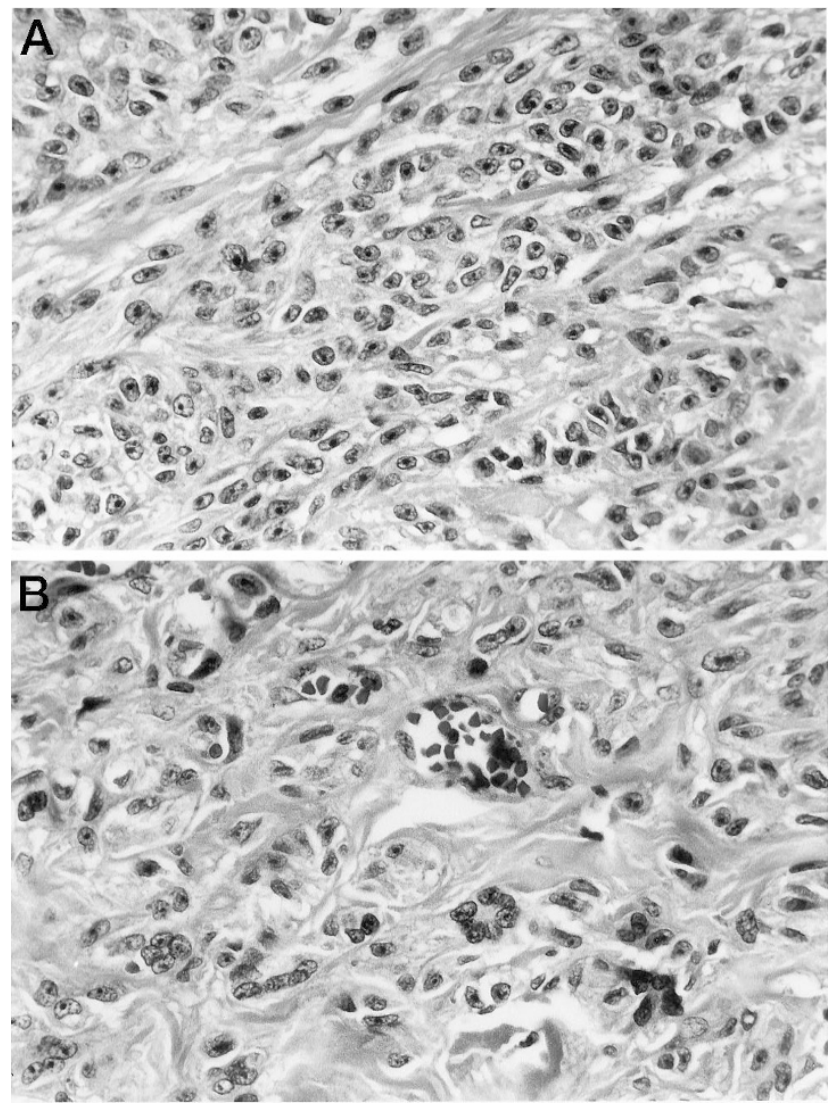

FIGURE 1. Clear cell sarcoma. A mixture of spindled and epithelioid cells have open chromatin and prominent nucleoli. There is a vague nesting pattern (A). Other areas show floret-like multinucleated giant cells, a helpful diagnostic clue (B) (Patient 7).

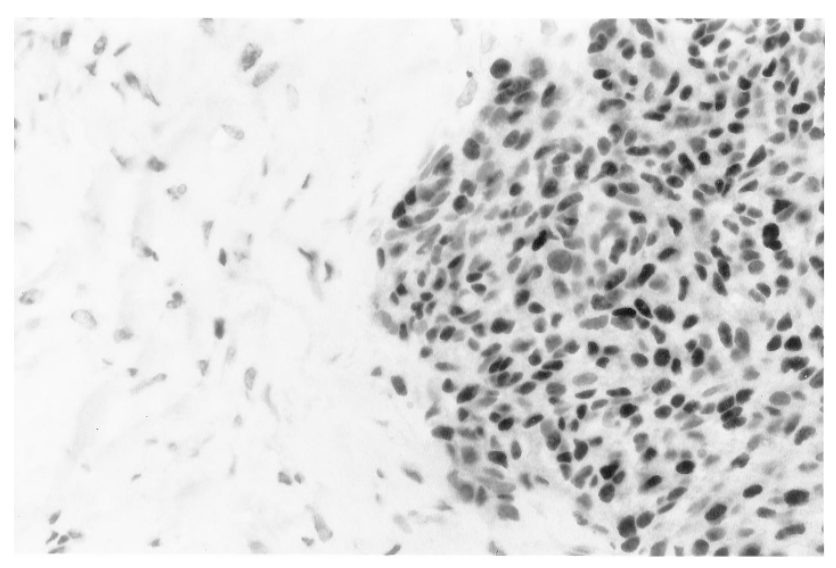

FIGURE 2. Clear cell sarcoma. Strong nuclear staining for microphthalmia transcription factor is present in this tumor. Stromal cells adjacent to the tumor are nonreactive (Patient 7).

sociation with tendon, fascia, or aponeuroses $(1,2)$. Several lines of evidence point to melanocytic differentiation in clear cell sarcoma-hence the alternative designation "melanoma of soft parts." In many of these tumors, melanin pigment is appreciated on routine light microscopy. Furthermore, electron microscopic examination demonstrates melanosomes in most tumors (3-5). Immunoreac- tivity for HMB-45, an antibody that recognizes the melanosome matrix protein pmel 17 or gp100 and is relatively specific for melanocytic lesions, is usually seen.

Although many features point to melanocytic differentiation, analysis of other data suggests clear cell sarcoma is a distinctive tumor, not simply a conventional-type melanoma that arises in soft tissue. In addition to its distinctive clinical and histopathologic features, clear cell sarcoma shows a consistent and distinctive translocation between chromosomes 12 and 22 resulting in fusion of the EWS gene and ATF-1 (6-9). The presence of this unique cytogenetic aberration, which has never been described in conventional malignant melanoma, argues for the classification of clear cell sarcoma as a distinct entity.

Compared with conventional melanoma, clear cell sarcoma tends to have a deeper location and to have more uniform cytologic features; in addition, it often contains distinctive multinucleated giant cells. Often, however, it is difficult to distinguish large spindle cell melanomas that lack a demonstrable in situ component from clear cell sarcoma on the basis of histologic examination alone. Unfortunately, there are no currently used immunomarkers that allow this distinction to be made. We studied the immunoreactivity of clear cell sarcoma for Mitf after encountering several tumors that were nonreactive for the D5 antibody in hopes that it might allow for this distinction. Mitf, the protein encoded by the $m i$ gene, has been shown to be essential for the development and survival of melanocytes and is thought to represent a central transcriptional regulator of enzymes involved in melanin biosynthesis. Like HMB-45, Mitf-D5 recognizes a melanocyte antigen and shows reactivity for benign melanocytes (nevus cells and normal skin melanocytes) and melanoma cells. Consistent D5 staining in amelanotic epithelioid melanomas also suggests that factors downstream from Mitf downregulate pigment production (10).

The monoclonal anti-Mitf antibody D5, generated against the $N$ terminus of human microphthalmia protein, was recently shown to be sensitive for melanoma diagnosis (10). In the latter series of 76 consecutively accessioned melanomas, all patients showed nuclear reactivity for the D5 antibody. Furthermore, S-100 and HMB-45 failed to detect five and seven cases, respectively, of the 76 melanomas that were immunoreactive for D5. The results of this same study also suggested that Mitf is a specific marker for melanocytic differentiation. None of the 60 nonmelanomas studied showed nuclear staining. Cytoplasmic staining, which is not considered to be a positive result, was seen in two patients with breast carcinoma. 
Because a significant fraction of clear cell sarcomas, particularly metastatic lesions and those that involve the superficial dermis, may be difficult to distinguish from melanoma on simple morphologic grounds, a marker that facilitates this distinction would be welcome. We began our study hoping that D5 would distinguish clear cell sarcoma from melanoma. The results of our study show that D5 does not accomplish this task. In fact, the $75 \%$ sensitivity of D5 as a marker of clear cell sarcoma is greater than the $26 \%$ sensitivity we found for spindle cell and desmoplastic melanomas (11). This comparison is important because melanomas with spindle cell cytomorphology are the most likely lesions to be confused with clear cell sarcoma. Although D5 does not aid in the distinction of clear cell sarcoma from melanoma, these findings are nevertheless important in lending further support for melanocytic differentiation in clear cell sarcoma. We are aware of one report in abstract form that reports similar staining of clear cell sarcoma with D5. Koch et al. (12) recently showed that 3 of 9 (33\%) of clear cell sarcomas investigated were immunoreactive for D5.

It should be emphasized that only nuclear reactivity is considered a positive result. A faint cytoplasmic blush was seen in samples from several patients who showed nuclear reactivity for D5. In our experience, faint cytoplasmic staining is often seen in melanoma and is considered nonspecific. Strong D5 cytoplasmic positivity was not seen in any clear cell sarcomas in this series. Likewise, we have not seen strong cytoplasmic positivity in any melanomas. Strong cytoplasmic staining (in the absence of nuclear staining) has been identified in breast carcinoma (10).

In conclusion, the majority of clear cell sarcomas are immunoreactive for D5, an antibody against Mitf. D5 is therefore not a useful marker for distinguishing melanoma from clear cell sarcoma. Immunoreactivity for D5 in clear cell sarcoma further supports the hypothesis that this is a tumor demonstrating true melanocytic differentiation. It is possible that Mitf expression plays a role in cell survival (13). If so, a more complete understanding of its biochemical activities may lead to the exploitation of Mitf as a therapeutic target. More study is needed to further delineate the sensitivity and specificity of the D5 antibody.

\section{REFERENCES}

1. Enzinger FM. Clear cell sarcoma of tendons and aponeuroses: an analysis of 21 cases. Cancer 1968;18:1163-74.

2. Chung EB, Enzinger FM. Malignant melanoma of soft parts. A reassessment of clear cell sarcoma. Am J Surg Pathol 1983;7:403-13.

3. Kindblom L-G, Lodding P, Angervall L. Clear cell sarcoma of tendons and aponeuroses. An immunohistochemical and electron microscopic analysis indicating neural crest origin. Virchows Arch 1983;401:109-28.

4. Benson JD, Kraemer B, Mackay B. Malignant melanoma of soft parts: an ultrastructural study of four cases. Ultrastruct Pathol 1985;8:57-70.

5. Hasegawa T, Hirose T, Kudo E, Hizawa K. Clear cell sarcoma: an immunohistochemical and ultrastructural study. Acta Pathol Jpn 1989;39:321-7.

6. Bridge JA, Borek DA, Neff JR, Huntrakoon M. Chromosomal abnormalities in clear cell sarcoma: implications for histogenesis. Am J Clin Pathol 1990;93:26-31.

7. Bridge JA, Sreekantaiah C, Neff JR, Sandberg AA. Cytogenetic findings in clear cell sarcoma of tendons and aponeuroses: malignant melanoma of soft parts. Cancer Genet Cytogenet 1991;52:101-6.

8. Travis JA, Bridge JA. Significance of both numerical and structural chromosomal abnormalities in clear cell sarcoma. Cancer Genet Cytogenet 1992;64:104-6.

9. Zucman J, Delattre O, Desmase C, et al. EWS and ATF-1 gene fusion induced by $\mathrm{t}(12 ; 22)$ in malignant melanoma of soft parts. Nat Genet 1993;4:341-5.

10. King R, Weilbaecher KN, McGill G, Cooley E, Mihm M, Fisher DE. Micropthalmia transcription factor: a sensitive and specific marker for melanoma diagnosis. Am J Pathol 1999;155: 731-8.

11. Granter SR, Weilbaecher KN, Fletcher CDM, Quigley C, Fisher DE. Microphthalmia transcription factor is not a sensitive marker for the diagnosis of desmoplastic melanoma and spindle cell (non-desmoplastic) melanoma. In preperation.

12. Koch MB, Arbiser ZK, Weiss SW, Shih I-H, Folpe AL. Melanoma cell adhesion molecule (Mel-CAM, CD146) and microphthalmia transcription factor (MiTF) expression distinguish desmoplastic sarcomatoid melanoma from morphologic mimics [abstract]. Mod Pathol 2000;13:63A.

13. Lerner AB, Shibahara T, Boissy RE, Jacobson KA, Lamoreuk ML, Moellmann GE. A mouse model for vitiligo. J Invest Dermatol 1986;87:299-304. 